\title{
Sundman Symmetries of Nonlinear Second-Order and Third-Order Ordinary Differential Equations
}

Norbert EULER and Marianna EULER

Department of Mathematics, Luleå University of Technology, SE-971 87 Luleå, Sweden

E-mails: Norbert@sm.luth.se, Marianna@sm.luth.se

\begin{abstract}
We investigate the Sundman symmetries of second-order and third-order nonlinear ordinary differential equations. These symmetries, which are in general nonlocal transformations, arise from generalised Sundman transformations of autonomous equations. We show that these transformations and symmetries can be calculated systematically and can be used to find first integrals of the equations.
\end{abstract}

\section{Introduction}

In the general classification of linearisable third-order ordinary differential equations under the generalised Sundman transformation

$$
X=F(x, t), \quad d T=G(x, t) d t, \quad G \frac{\partial F}{\partial x} \neq 0,
$$

we [5] observed that for every $F$ and $G$ in (1.1) that linearises a given third-order equation to $X^{\prime \prime \prime}=0$ there exists another $F$ and $G$ which also results in the linearisation of the equation, notably

$$
F \rightarrow \frac{1}{F}, \quad G \rightarrow \frac{G}{F^{3 / 2}} .
$$

This means that every third-order equation

$$
\dddot{x}=E(t, x, \dot{x}, \ddot{x})
$$

which can be linearised to $X^{\prime \prime \prime}=0$ under (1.1) is invariant under the transformation

$$
\begin{aligned}
& F(\tilde{x}, \tilde{t})=F(x, t)^{-1} \\
& G(\tilde{x}, \tilde{t}) d \tilde{t}=F(x, t)^{-3 / 2} G(x, t) d t
\end{aligned}
$$


for a given $F$ and $G$ that achieves this linearisation. We named the transformation (1.2)(1.3) the Sundman symmetry [5] of linearisable third-order equations.

Note that the generalised Sundman transformation (1.1) is a more general transformation than a point or contact transformation in the sense that (1.1) contains a nonlocal term of the form

$$
T=\int G(x(t), t) d t
$$

We refer to the book [10] for examples and methods relating to point transformations of differential equations.

Remark: Throughout this article overdot and prime refer to ordinary derivatives of the dependent variables and subscripts refer to partial derivatives.

We give a simple pedagogical example to demonstrate the generalised Sundman transformation and the Sundman symmetry for a linearisable equation:

Introductory example: Consider the equation

$$
\dddot{x}-x^{-1} \dot{x} \ddot{x}=0,
$$

which can be linearised to $X^{\prime \prime \prime}=0$ by the generalised Sundman transformation

$$
X=x^{2}, \quad d T=x d t
$$

and by (1.2)-(1.3) equation (1.4) admits the Sundman symmetry

$$
\tilde{x}=x^{-1}, \quad \tilde{t}=\int x^{-1} d t .
$$

We then use the generalised Sundman transformation (1.5) and transform the first integrals of $X^{\prime \prime \prime}=0$,

$$
I_{1}=X^{\prime \prime}, \quad I_{2}=X X^{\prime \prime}-\frac{1}{2}\left(X^{\prime}\right)^{2}
$$

to obtain the first integrals of (1.4), namely

$$
\tilde{I}_{1}=\frac{\ddot{x}}{x}, \quad \tilde{I}_{2}=x \ddot{x}-\dot{x}^{2} .
$$

Note that the Sundman symmetry (1.6) transforms solutions of (1.4) to solutions of (1.4). For example the solution $x=t$ transforms to the solution $x=c_{1} \exp (-t)$, where $c_{1}$ is an arbitrary constant namely the constant of integration in (1.6b). We point out that Corollary 4.2 of section 4.2 gives the most general Sundman symmetry which a thirdorder linearisable ordinary differential equation may admit namely (4.27) and (4.28). In Example 4.3 of section 4.2 we show that the Sundman symmetry (4.27) - (4.28) transforms the particular solution $x=t$ to the general solution of (1.4), namely

$$
x=\beta_{2} e^{-\beta_{1} t}+\beta_{3} e^{\beta_{1} t},
$$

where $\beta_{j}$ are arbitrary constants. 
As part of this introductory example we show that the general solution of (1.4) can also be obtained by transforming a particular solution of $X^{\prime \prime \prime}=0$ under the generalised Sundman transformation (1.5). We use

$$
X(T)=c_{1}^{2} T^{2}+c_{3}, \quad c_{1}, c_{3}: \text { arbitrary constants. }
$$

By (1.5) we have $x=\sqrt{X}$ (we use the positive sign), so that

$$
T=\frac{1}{c_{1}}\left(x^{2}-c_{3}\right)^{1 / 2}
$$

and

$$
\int\left(c_{1}^{2} T^{2}+c_{3}\right)^{-1 / 2} d T=t+\frac{1}{c_{1}} \ln |a|,
$$

where $a$ is a constant of integration. We perform the integral (1.9) and use (1.8) to eliminate $T$. We then obtain the general solution of (1.4), namely

$$
x(t)=\frac{a}{2} \exp \left(c_{1} t\right)-\frac{c_{3}}{2 a} \exp \left(-c_{1} t\right) .
$$

To end this example we mention that the Sundman symmetry (1.6) transforms first integrals of (1.4) to first integrals of (1.4): $\tilde{I}_{1}$ transforms to $\tilde{I}_{2}$ and $\tilde{I}_{2}$ to $\tilde{I}_{1}$.

Our aim in the present article is to investigate the Sundman symmetry of second-order and third-order nonlinearisable ordinary differential equations (although we also include the linearisable cases), that is to calculate Sundman symmetries of equations which are not necessarily linearisable by a generalised Sundman transformation. We show that Sundman symmetries exist for a large class of nonlinear equations and can systematically be calculated. We furthermore show that these symmetries can be used effectively to obtain first integrals of the equations and transform solutions to more general solutions (in some cases the general solutions). We point out that the first integrals can in some cases be integrated (perhaps parametrically) to obtain the general (parametric) solutions. It is not our aim to perform parameric calculations here, but we give one such example as part of Example 3.1.

\section{General considerations}

In correspondence with [5] we define the generalised Sundman transformation and the Sundman symmetry of an $n$th order ordinary differential equation

$$
x^{(n)}=E_{1}\left(t, x, \dot{x}, \ddot{x}, \dddot{x}, \ldots, x^{(n-1)}\right),
$$

where $x=x(t)$.

Definition 2.1 The generalised Sundman transformation of equation (2.1) is a coordinate transformation of the form (1.1), that is

$$
X(T)=F(x(t), t), \quad d T=G(x(t), t) d t, \quad \frac{\partial F}{\partial x} \neq 0, G \neq 0,
$$


for which the differentiable functions $F$ and $G$ are determined such that (2.1) can be transformed in the autonomous equation

$$
X^{(n)}=E_{0}\left(X, X^{\prime}, X^{\prime \prime}, X^{\prime \prime \prime}, \ldots, X^{(n-1)}\right),
$$

where $X=X(T)$. If (2.2) is linear, then (2.1) is said to be linearisable.

Definition 2.2 A Sundman symmetry for equation (2.1) is a transformation of the form

$$
F(\tilde{x}, \tilde{t})=P(F(x, t), G(x, t)), \quad G(\tilde{x}, \tilde{t}) d \tilde{t}=Q(F(x, t), G(x, t)) d t
$$

which keeps (2.1) invariant for some differentiable functions $P$ and $Q$, i.e. (2.1) is transformed to

$$
\tilde{x}^{(n)}=E_{1}\left(\tilde{t}, \tilde{x}, \dot{\tilde{x}}, \ddot{\tilde{x}}, \dddot{\tilde{x}}, \ldots, \tilde{x}^{(n-1)}\right),
$$

whereby $F$ and $G$ are functions in (1.1) which transform (2.1) to (2.2) (see Diagram 1). A Sundman symmetry (2.3) is called trivial if $P(F, G)=F$ and $Q(F, G)=G$.

For a given autonomous equation (2.2) the generalised Sundman transformation (1.1) leads to a set of differential conditions on $F$ and $G$ by which (2.1) can be transformed to (2.2). We call these conditions the Sundman determining equations.

A Sundman symmetry (2.3) can then be obtained by choosing $P$ and $Q$ such that the Sundman determining equations remain invariant. If

$$
X=F(\tilde{x}, \tilde{t}), \quad d T=G(\tilde{x}, \tilde{t}) d \tilde{t}
$$

transforms (2.4) to (2.2) and

$$
X=P(F(x, t), G(x, t)), \quad d T=Q(F(x, t), G(x, t)) d t
$$

also transforms the same equation (2.1) to (2.2), then the composition of these two generalised Sundman transformations leads to the Sundman symmetry (2.3) for (2.1) (see Diagram 1). The choice $P=F$ and $Q=G$ corresponds to the trivial Sundman symmetry.

If, for example,

$$
F(\tilde{x}, \tilde{t})=\tilde{x}, \quad G(\tilde{x}, \tilde{t})=1,
$$

then the generalised Sundman transformation (1.1) is just the identity transformation $(X=\tilde{x}, T=\tilde{t})$ and equation (2.4) has the exact same form as equation (2.2). The Sundman symmetry of $(2.1)$ then takes the form

$$
\tilde{x}=P(x), \quad \tilde{t}=\int Q(x) d t .
$$

In fact a Sundman symmetry constructed in this way (from the identity) plays an important role in the calculation of first integrals as is discussed in Section 4. Instead of 


\section{Diagram 1}

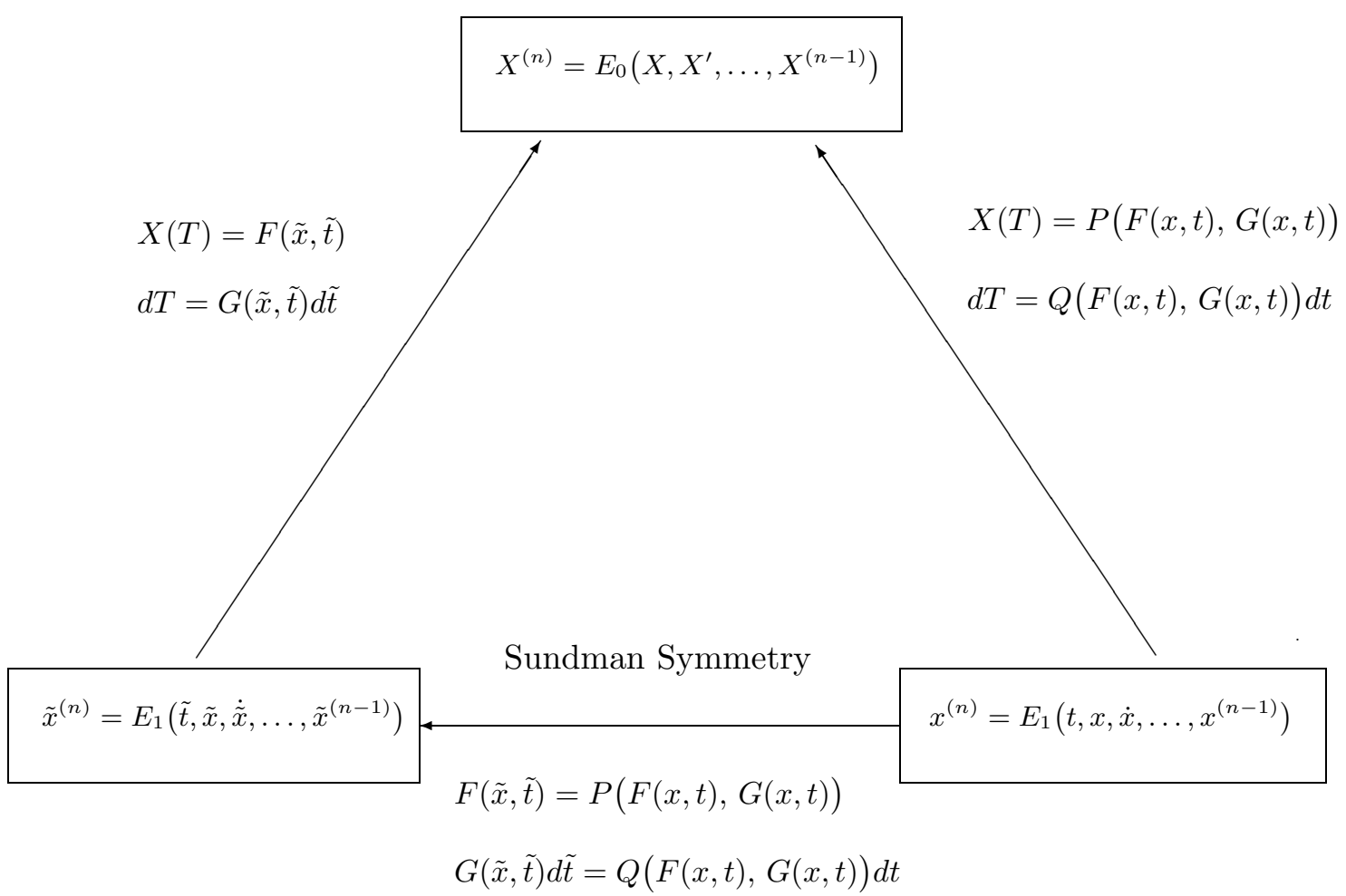

the identity one can also consider other forms of $F$ and $G$ that correspond to transformations which keep (2.2) invariant such as discrete transformations (see e.g. [6], [7] and [8] for a method to find discrete symmetries of ordinary differential equations by the use of Lie symmetry algebras). If the Sundman symmetry (2.3) is nontrivial, then every given (discrete) transformation which keeps (2.2) invariant leads to a nontrivial Sundman symmetry for (2.2) for corresponding $F$ and $G$ in (1.1).

\section{Sundman symmetries for second-order equations}

We consider the following class of second-order autonomous equations

$$
X^{\prime \prime}+a_{2}(X)\left(X^{\prime}\right)^{2}+a_{1}(X) X^{\prime}+a_{0}(X)=0,
$$

where $a_{2}, a_{1}$ and $a_{0}$ are differentiable functions which are to be determined such that (3.1) leads to a class of equations with nontrivial Sundman symmetries. We distinguish between three cases given respectively by Proposition 3.1, Proposition 3.2 and Proposition 3.3 below.

Proposition 3.1: Consider (3.1) with both $a_{1} \neq 0$ and $a_{2} \neq 0$, that is

$$
X^{\prime \prime}+a_{2}(X)\left(X^{\prime}\right)^{2}+a_{1}(X) X^{\prime}+a_{0}(X)=0 .
$$

The equation

$$
\ddot{x}+\Lambda_{2} \dot{x}^{2}+\Lambda_{1} \dot{x}+\Lambda_{0}=0,
$$


which can be transformed to (3.1) under (1.1), where

$$
\begin{aligned}
& \Lambda_{2}(F, G ; x, t)=\frac{F_{x x}}{F_{x}}-\frac{G_{x}}{G}+a_{2}(F) \\
& \Lambda_{1}(F, G ; x, t)=2 \frac{F_{x t}}{F_{x}}-\frac{G_{x}}{G} \frac{F_{t}}{F_{x}}-\frac{G_{t}}{G}+a_{1}(F) G+2 a_{2}(F) F_{t} \\
& \Lambda_{0}(F, G ; x, t)=\frac{F_{t t}}{F_{x}}-\frac{G_{t}}{G} \frac{F_{t}}{F_{x}}+a_{1}(F) \frac{G F_{t}}{F_{x}}+a_{2}(F) \frac{F_{t}^{2}}{F_{x}}+a_{0}(F) \frac{G^{2}}{F_{x}},
\end{aligned}
$$

admits a Sundman symmetry of the form (2.3) if and only if $P$ and $Q$ are given by

$$
P=P(F), \quad Q=G \Psi(F),
$$

where $P, \Psi, a_{2}, a_{1}$ and $a_{0}$ satisfy the following conditions:

$$
\begin{aligned}
& a_{1}(X)=a_{2}(X) \neq 0 \\
& a_{1}(P) \Psi(F)=a_{1}(F) \\
& a_{0}(P) \Psi^{2}(F)=a_{0}(F) \frac{d P}{d F} \\
& \Psi(F) \frac{d^{2} P}{d F^{2}}-\frac{d \Psi}{d F} \frac{d P}{d F}+a_{2}(P) \Psi(F)\left(\frac{d P}{d F}\right)^{2}-a_{2}(F) \Psi(F) \frac{d P}{d F}=0 .
\end{aligned}
$$

The Sundman symmetry of (3.2) is therefore of the form

$$
\begin{aligned}
& F(\tilde{x}, \tilde{t})=P(F(x, t)) \\
& G(\tilde{x}, \tilde{t}) d \tilde{t}=G(x, t) \Psi(F(x, t)) d t,
\end{aligned}
$$

where conditions (3.5)-(3.8) must be satisfied.

On the proof: By the generalised Sundman transformation (1.1) the equation (3.1) takes the form (3.2). The Sundman symmetry is then obtained by solving $P$ and $Q$ from the condition

$$
\begin{aligned}
& \Lambda_{2}(F, G ; x, t)=\Lambda_{2}(\hat{F}, \hat{G} ; x, t) \\
& \Lambda_{1}(F, G ; x, t)=\Lambda_{1}(\hat{F}, \hat{G} ; x, t) \\
& \Lambda_{0}(F, G ; x, t)=\Lambda_{0}(\hat{F}, \hat{G} ; x, t),
\end{aligned}
$$

where

$$
\begin{aligned}
& \hat{F}(x, t)=P(F(x, t), G(x, t)) \\
& \hat{G}(x, t)=Q(F(x, t), G(x, t)) .
\end{aligned}
$$

This leads to a set of differential conditions on $P$ and $Q$ which reduces to conditions (3.5)-(3.8). 
Example 3.1: We consider (3.1) with $a_{0}(X)=a_{1}(X)=a_{2}(X)=X$, that is

$$
X^{\prime \prime}+X\left(X^{\prime}\right)^{2}+X X^{\prime}+X=0 .
$$

By Proposition 3.1 we find that (3.11) results in a Sundman symmetry of the form (3.9)(3.10) with

$$
P=\sqrt{F^{2}+1}, \quad \Psi=\frac{F}{\sqrt{F^{2}+1}} .
$$

Thus

$$
F(\tilde{x}, \tilde{t})=\sqrt{F^{2}(x, t)+1}, \quad G(\tilde{x}, \tilde{t}) d \tilde{t}=\frac{G(x, t) F(x, t)}{\sqrt{F^{2}(x, t)+1}} d t
$$

is the Sundman Symmetry for the equation

$$
\begin{aligned}
\ddot{x}+ & \left(\frac{F_{x x}}{F_{x}}-\frac{G_{x}}{G}+F\right) \dot{x}^{2}+\left(2 \frac{F_{x t}}{F_{x}}-\frac{G_{x}}{G} \frac{F_{t}}{F_{x}}-\frac{G_{t}}{G}+F G+2 F F_{t}\right) \dot{x} \\
& +\frac{F_{t t}}{F_{x}}-\frac{G_{t}}{G} \frac{F_{t}}{F_{x}}+\frac{G F F_{t}}{F_{x}}+\frac{F F_{t}^{2}}{F_{x}}+\frac{F G^{2}}{F_{x}}=0,
\end{aligned}
$$

where $F$ and $G$ are those functions in the generalised Sundman transformation (1.1) which transforms (3.12) to (3.11). Let

$$
F(x, t)=x, \quad G(x, t)=1,
$$

i.e. the identity transformation $(X=x, T=t)$. Then the the autonomous equation (3.11) transforms to itself, i.e.

$$
\ddot{x}+x \dot{x}^{2}+x \dot{x}+x=0,
$$

and the Sundman symmetry for (3.13) takes the form

$$
\tilde{x}=\sqrt{x^{2}+1}, \quad \tilde{t}=\int \frac{x}{\sqrt{x^{2}+1}} d t .
$$

Let, for example,

$$
F(x, t)=x, \quad G(x, t)=x t .
$$

Then (3.12) takes the form

$$
\ddot{x}+\left(\frac{x^{2}-1}{x}\right) \dot{x}^{2}+\left(\frac{x^{2} t^{2}-1}{t}\right) \dot{x}+x^{3} t^{2}=0
$$

for which the Sundman symmetry is then

$$
\tilde{x}=\sqrt{x^{2}+1}, \quad \tilde{t}=\sqrt{2}\left(\int \frac{x^{2} t}{x^{2}+1} d t\right)^{1 / 2} .
$$


The first integral of (3.11) can easily be calculated by the method described in [4]. We obtain

$$
I_{1}=\frac{1}{2} X^{2}+\frac{1}{2} \ln \left(\left(X^{\prime}+\frac{1}{2}\right)^{2}+\frac{3}{4}\right)-\frac{1}{\sqrt{3}} \tan ^{-1}\left(\frac{2}{\sqrt{3}}\left(X^{\prime}+\frac{1}{2}\right)\right) .
$$

A $t$-dependent first integral of (3.14) is then obtained by transforming (3.15) by the generalised Sundman transformation

$$
X=x, \quad d T=x t d t .
$$

The first integral, (3.15), can be integrated parametrically by the following method. Consider a first-order equation of the form

$$
H\left(x(t), \frac{d x}{d t}\right)=0 .
$$

Let $x(t)=f(\tau), d x / d t=g(\tau), \tau=\tau(t)$, where $f$ and $g$ satisfy the algebraic relation

$$
H(f(\tau), g(\tau))=0
$$

and $\tau$ is a parameter. By

$$
\frac{d x}{d t}=\frac{d f}{d \tau} \frac{d \tau}{d t}
$$

it follows that

$$
g(\tau)=\frac{d f}{d \tau} \frac{d \tau}{d t}, \quad \text { i.e. } \quad \int d t=\int \frac{d f(\tau)}{d \tau} \frac{1}{g(\tau)} d \tau+C,
$$

where $C$ is a constant of integration. The general parametric solution of (3.17) is then

$$
\begin{aligned}
& x(\tau)=f(\tau) \\
& t(\tau)=\int \frac{d f(\tau)}{d \tau} \frac{1}{g(\tau)} d \tau+C \\
& H(f(\tau), g(\tau))=0
\end{aligned}
$$

By the method outlined above we integrate the first integral (3.15) parametrically to obtain the general solution of (3.11) in the form

$$
\begin{aligned}
& X(\tau)=\left[2 I_{1}-\ln \left(\tau^{2}+\frac{3}{4}\right)+\frac{2}{\sqrt{3}} \tan ^{-1}\left(\frac{2}{\sqrt{3}} \tau\right)\right]^{1 / 2} \\
& T(\tau)=C_{1}-\frac{4}{\sqrt{3}} \tan ^{-1}\left(\frac{2}{\sqrt{3}} \tau\right),
\end{aligned}
$$

where $\tau \in \Re$ is a parameter and $I_{1}, C_{1}$ are two arbitrary constants. The solution to (3.14) is then obtained by the transformation (3.16) with

$$
d T=\frac{d T}{d \tau} d \tau
$$


and by the parametric solution (3.19) and (3.20). We obtain the general solution of (3.14) in parametric form as

$$
\begin{aligned}
& x(\tau)=X(\tau) \\
& t(\tau)^{2}=-2 \int\left(\frac{8\left[2 I_{1}-\ln \left(\tau^{2}+3 / 4\right)+(2 / \sqrt{3}) \tan ^{-1}(2 \tau / \sqrt{3})\right]^{-1 / 2}}{4 \tau^{2}+3}\right) d \tau+C_{2},
\end{aligned}
$$

where $\tau \in \Re$ is a parameter and $I_{1}, C_{2}$ are two arbitrary constants.

We give the following two Propositions without further comments on their proofs:

Proposition 3.2: Consider (3.1) with $a_{1}=0$, that is

$$
X^{\prime \prime}+a_{2}(X)\left(X^{\prime}\right)^{2}+a_{0}(X)=0 .
$$

The equation

$$
\ddot{x}+\Lambda_{2} \dot{x}^{2}+\Lambda_{1} \dot{x}+\Lambda_{0}=0,
$$

which can be transformed to (3.21) under (1.1), where

$$
\begin{aligned}
& \Lambda_{2}(F, G ; x, t)=\frac{F_{x x}}{F_{x}}-\frac{G_{x}}{G}+a_{2}(F) \\
& \Lambda_{1}(F, G ; x, t)=2 \frac{F_{x t}}{F_{x}}-\frac{G_{x}}{G} \frac{F_{t}}{F_{x}}-\frac{G_{t}}{G}+2 a_{2}(F) F_{t} \\
& \Lambda_{0}(F, G ; x, t)=\frac{F_{t t}}{F_{x}}-\frac{G_{t}}{G} \frac{F_{t}}{F_{x}}+a_{2}(F) \frac{F_{t}^{2}}{F_{x}}+a_{0}(F) \frac{G^{2}}{F_{x}},
\end{aligned}
$$

admits a Sundman symmetry of the form (2.3) if and only if $P$ and $Q$ are given by

$$
P=P(F), \quad Q=G \Psi(F)
$$

and $P, \Psi, a_{2}$ and $a_{0}$ satisfy the following conditions:

$$
\begin{aligned}
& a_{0}(P(F)) \Psi^{2}(F)=a_{0}(F) \frac{d P}{d F} \\
& \Psi(F) \frac{d^{2} P}{d F^{2}}-\frac{d \Psi}{d F} \frac{d P}{d F}+a_{2}(P(F)) \Psi(F)\left(\frac{d P}{d F}\right)^{2}-a_{2}(F) \Psi(F) \frac{d P}{d F}=0 .
\end{aligned}
$$

The Sundman symmetry of (3.22) is therefore of the form

$$
\begin{aligned}
& F(\tilde{x}, \tilde{t})=P(F(x, t)) \\
& G(\tilde{x}, \tilde{t}) d \tilde{t}=G(x, t) \Psi(F(x, t)) d t,
\end{aligned}
$$

where conditions (3.25) and (3.26) must be satisfied. 
Proposition 3.3: Consider (3.1) with $a_{2}=0$, that is

$$
X^{\prime \prime}+a_{1}(X) X^{\prime}+a_{0}(X)=0 .
$$

The equation

$$
\ddot{x}+\Lambda_{2} \dot{x}^{2}+\Lambda_{1} \dot{x}+\Lambda_{0}=0,
$$

which can be transformed to (3.29) under (1.1), where

$$
\begin{aligned}
& \Lambda_{2}(F, G ; x, t)=\frac{F_{x x}}{F_{x}}-\frac{G_{x}}{G} \\
& \Lambda_{1}(F, G ; x, t)=2 \frac{F_{x t}}{F_{x}}-\frac{G_{x}}{G} \frac{F_{t}}{F_{x}}-\frac{G_{t}}{G}+a_{1}(F) G \\
& \Lambda_{0}(F, G ; x, t)=\frac{F_{t t}}{F_{x}}-\frac{G_{t}}{G} \frac{F_{t}}{F_{x}}+a_{1}(F) \frac{G F_{t}}{F_{x}}+a_{0}(F) \frac{G^{2}}{F_{x}}
\end{aligned}
$$

admits a Sundman symmetry of the form (2.3) if and only if

$$
\begin{aligned}
& P=P(F), \quad Q=G \frac{d P}{d F} \\
& a_{0}(F)=\lambda a_{1}(F) \\
& a_{1}(P) \frac{d P}{d F}=a_{1}(F) .
\end{aligned}
$$

Here $\lambda$ is an arbitrary nonzero constant and $P$ an arbitrary differentiable function of $F$. The Sundman symmetry of (3.30) is therefore of the form

$$
\begin{aligned}
& F(\tilde{x}, \tilde{t})=P(F(x, t)) \\
& G(\tilde{x}, \tilde{t}) d \tilde{t}=G(x, t) \frac{d P}{d F}(F(x, t)) d t,
\end{aligned}
$$

where (3.33) and (3.34) must be satisfied.

Example 3.2: By Proposition 3.2 we find that

$$
X^{\prime \prime}-X^{-1}\left(X^{\prime}\right)^{2}-X^{2}=0
$$

results in a Sundman symmetry of the form (3.27)-(3.28) with

$$
P=c_{2}\left(c_{2} F+c_{1}\right), \quad \Psi=\frac{F}{c_{2} F+c_{1}},
$$

where $c_{1} \in \Re$ and $c_{2} \in \Re \backslash 0$.

Example 3.3: By Proposition 3.3 we find that

$$
X^{\prime \prime}+\lambda_{1} X^{n} X^{\prime}+\lambda_{2} X^{n}=0
$$


results in a Sundman symmetry of the form (3.35)-(3.36) with

$$
P(F)=\left(F^{n+1}+c_{1}\right)^{1 /(n+1)}
$$

where $n \in \mathcal{Q} \backslash\{-1\}, \lambda_{1}, \lambda_{2} \in \Re \backslash 0$ and $c_{1} \in \Re$.

The second-order linearisable case: By Proposition 3.3 we have

Corollary 3.1: Every second-order ordinary differential equation which can be transformed to

$$
X^{\prime \prime}=0
$$

under the generalised Sundman transformation (1.1) admits the Sundman symmetry (3.35)(3.36), that is

$$
\begin{aligned}
& F(\tilde{x}, \tilde{t})=P(F(x, t)) \\
& G(\tilde{x}, \tilde{t}) d \tilde{t}=G(x, t) \frac{d P(F(x, t))}{d F} d t,
\end{aligned}
$$

with no further conditions on the differentiable function $P$.

Example 3.4: Consider the second-order equation

$$
\ddot{x}-\frac{2}{x} \dot{x}^{2}+\frac{2}{t^{2}} x=0 .
$$

Duarte et al [3] showed that (3.38) can be linearised to (3.37) by the generalised Sundman transformation

$$
X=t^{3} x^{3 / 2}, \quad d T=t x^{5 / 2} d t .
$$

By Proposition 3.3 the Sundman symmetry for (3.38) is of the form (3.35)-(3.36) with $F=t^{3} x^{3 / 2}$ and $G=t x^{5 / 2}$, which may be written as

$$
\tilde{t}=\left(c-3 \int \frac{d P}{d F} P^{-5 / 3} t x^{5 / 2} d t\right)^{-1 / 3}, \quad \tilde{x}=P^{2 / 3}\left(c-3 \int \frac{d P}{d F} P^{-5 / 3} t x^{5 / 2} d t\right)^{2 / 3},
$$

where $P=P(F)$ is an arbitrary function and $c$ an arbitrary constant. Let, for example, $P(F)=F^{-1}$. Then the Sundman symmetry takes the form

$$
\tilde{t}=\left(c-3 \int x^{2} d t\right)^{-1 / 3}, \quad \tilde{x}=t^{-2} x^{-1}\left(c-3 \int x^{2} d t\right)^{2 / 3} .
$$

Finally we map the special solution of (3.37) to the general solution of (3.38) by (3.39). Consider the following special solution of (3.37):

$$
X(T)=c_{1} T .
$$

The generalised Sundman transformation (3.39) can be written in the form

$$
X=t^{3} x^{3 / 2}, \quad X^{-5 / 3} d T=t^{-4} d t
$$


which by invoking (3.40) the second part becomes

$$
\begin{aligned}
& T=\frac{1}{c_{1}} t^{3} x^{3 / 2} \\
& \int\left(c_{1} T\right)^{-5 / 3} d T=-\frac{1}{3} t^{-3}+\frac{2 c_{1}}{9} a,
\end{aligned}
$$

where $a$ is a constant of integration. The general solution of (3.38) follows, namely

$$
x(t)=\frac{9}{2 c_{1}} \frac{t}{a t^{3}+1} .
$$

We mention that (3.38) can also be linearised to (3.37) by the point transformation [3]

$$
X(T)=x t^{2}, \quad T=\frac{x}{t} .
$$

\section{Sundman symmetries for third-order equations}

We consider the following class of third-order autonomous equations

$$
\begin{aligned}
X^{\prime \prime \prime} & +a_{5}(X) X^{\prime \prime}+a_{4}(X) X^{\prime} X^{\prime \prime}+a_{3}(X)\left(X^{\prime}\right)^{3}+a_{2}(X)\left(X^{\prime}\right)^{2} \\
& +a_{1}(X) X^{\prime}+a_{0}(X)=0
\end{aligned}
$$

where $a_{j}(j=0,1, \ldots, 5)$ are differentiable functions which are to be determined such that (4.1) admit nontrivial Sundman symmetries. For this purpose we have the following

Proposition 4.1: Equation

$$
\begin{aligned}
\dddot{x}+ & \Lambda_{5}(x, t) \ddot{x}+\Lambda_{4}(x, t) \dot{x} \ddot{x}+\Lambda_{3}(x, t) \dot{x}^{3}+\Lambda_{2}(x, t) \dot{x}^{2} \\
& +\Lambda_{1}(x, t) \dot{x}+\Lambda_{0}(x, t)=0
\end{aligned}
$$

which can be transformed to (4.1) under (1.1), where

$$
\begin{aligned}
& \Lambda_{5}(x, t)=3 \frac{F_{x t}}{F_{x}}-3 \frac{G_{t}}{G}-\frac{F_{t}}{F_{x}} \frac{G_{x}}{G}+a_{4}(F) F_{t}+a_{5}(F) G \\
& \Lambda_{4}(x, t)=-4 \frac{G_{x}}{G}+3 \frac{F_{x x}}{F_{x}}+a_{4}(F) F_{x}
\end{aligned}
$$




$$
\begin{aligned}
& \Lambda_{3}(x, t)=\frac{F_{x x x}}{F_{x}}+3\left(\frac{G_{x}}{G}\right)^{2}-3 \frac{F_{x x}}{F_{x}} \frac{G_{x}}{G}-\frac{G_{x x}}{G}+a_{4}(F) F_{x x}-a_{4}(F) \frac{F_{x} G_{x}}{G} \\
& +a_{3}(F) F_{x}^{2} \\
& \Lambda_{2}(x, t)=3 \frac{F_{x x t}}{F_{x}}+3 \frac{F_{t}}{F_{x}}\left(\frac{G_{x}}{G}\right)^{2}-6 \frac{F_{x t}}{F_{x}} \frac{G_{x}}{G}-3 \frac{F_{x x}}{F_{x}} \frac{G_{t}}{G}+6 \frac{G_{x}}{G} \frac{G_{t}}{G} \\
& -\frac{F_{t}}{F_{x}} \frac{G_{x x}}{G}-2 \frac{G_{x t}}{G}-2 a_{4}(F) \frac{F_{t} G_{x}}{G}+3 a_{3}(F) F_{t} F_{x}-a_{4}(F) \frac{F_{x} G_{t}}{G} \\
& +a_{2}(F) F_{x} G+a_{5}(F) \frac{G F_{x x}}{F_{x}}+a_{4}(F) \frac{F_{t} F_{x x}}{F_{x}}+2 a_{4}(F) F_{x t}-a_{5}(F) G_{x} \\
& \Lambda_{1}(x, t)=3\left(\frac{G_{t}}{G}\right)^{2}+3 \frac{F_{x t t}}{F_{x}}-3 \frac{F_{t t}}{F_{x}} \frac{G_{x}}{G}-6 \frac{F_{x t}}{F_{x}} \frac{G_{t}}{G}+6 \frac{F_{t}}{F_{x}} \frac{G_{x}}{G} \frac{G_{t}}{G}-2 \frac{F_{t}}{F_{x}} \frac{G_{x t}}{G} \\
& -\frac{G_{t t}}{G}-2 a_{4}(F) \frac{F_{t} G_{t}}{G}-a_{4}(F) \frac{F_{t}^{2}}{F_{x}} \frac{G_{x}}{G}+2 a_{5}(F) \frac{G F_{x t}}{F_{x}}+a_{4}(F) F_{t t} \\
& -a_{5}(F) \frac{G_{x} F_{t}}{F_{x}}+2 a_{2}(F) F_{t} G+3 a_{3}(F) F_{t}^{2}-a_{5}(F) G_{t}+a_{1}(F) G^{2} \\
& +2 a_{4} \frac{F_{t} F_{x t}}{F_{x}} \\
& \Lambda_{0}(x, t)=-\frac{F_{t}}{F_{x}} \frac{G_{t t}}{G}-3 \frac{F_{t t}}{F_{x}} \frac{G_{t}}{G}+\frac{F_{t t t}}{F_{x}}+3 \frac{F_{t}}{F_{x}}\left(\frac{G_{t}}{G}\right)^{2}+a_{2}(F) \frac{G F_{t}^{2}}{F_{x}}+a_{3}(F) \frac{F_{t}^{3}}{F_{x}} \\
& -a_{5}(F) \frac{G_{t} F_{t}}{F_{x}}+a_{1}(F) \frac{G^{2} F_{t}}{F_{x}}+a_{5}(F) \frac{G F_{t t}}{F_{x}}+a_{4}(F) \frac{F_{t} F_{t t}}{F_{x}}+a_{0}(F) \frac{G^{3}}{F_{x}} \\
& -a_{4}(F) \frac{F_{t}^{2}}{F_{x}} \frac{G_{t}}{G}
\end{aligned}
$$

admits a Sundman symmetry of the form (2.3) if and only if $P$ and $Q$ are given by

$$
P=P(F), \quad Q=G \Psi(F)
$$


and $P, \Psi, a_{5}, a_{4}, a_{3}, a_{2}, a_{1}$ and $a_{0}$ satisfy the following conditions:

$$
\begin{aligned}
& a_{0}(F) \frac{d P}{d F}-a_{0}(P) \Psi^{3}=0 \\
& a_{1}(F)-a_{1}(P) \Psi^{2}=0 \\
& a_{5}(F)-a_{5}(P) \Psi=0 \\
& \frac{1}{3} a_{5}(F) \Psi^{-1} \frac{d \Psi}{d F}+a_{2}(P) \Psi \frac{d P}{d F}-\frac{1}{3} a_{5}(F) a_{4}(P) \frac{d P}{d F} \\
& \quad+\frac{1}{3} a_{4}(F) a_{5}(F)-a_{2}(F)=0 \\
& 3 \Psi \frac{d^{2} P}{d F^{2}}-4 \frac{d \Psi}{d F} \frac{d P}{d F}+a_{4}(P) \Psi\left(\frac{d P}{d F}\right)^{2}-a_{4}(F) \Psi \frac{d P}{d F}=0 \\
& \Psi^{-1} \frac{d^{2} \Psi}{d F^{2}}-\frac{5}{3} \Psi^{-2}\left(\frac{d \Psi}{d F}\right)^{2}-\frac{1}{3} a_{4}(F) \Psi^{-1} \frac{d \Psi}{d F}-\frac{d a_{4}(P)}{d P}\left(\frac{d P}{d F}\right)^{2} \\
& \quad-\frac{1}{3} a_{4}(P)^{2}\left(\frac{d P}{d F}\right)^{2}+\frac{d a_{4}(F)}{d F}+\frac{1}{3} a_{4}(F)^{2}-3 a_{3}(F)+3 a_{3}(P)\left(\frac{d P}{d F}\right)^{2}=0
\end{aligned}
$$

The Sundman symmetry of (4.2) is therefore of the form

$$
\begin{aligned}
& F(\tilde{x}, \tilde{t})=P(F(x, t)) \\
& G(\tilde{x}, \tilde{t}) d \tilde{t}=G(x, t) \Psi(F(x, t)) d t,
\end{aligned}
$$

where conditions (4.5)-(4.10) must be satisfied.

Example 4.1: Here we list some equations and the corresponding Sundman symmetries which were obtained by solving conditions (4.5)-(4.10) in Proposition 4.1:

a) The equation

$$
X^{\prime \prime \prime}-7 X^{-1} X^{\prime} X^{\prime \prime}+\lambda_{1} X^{2} X^{\prime}=0
$$

leads to a Sundman symmetry of the form (4.11)-(4.12) with

$$
P=F^{-1}, \quad \Psi=F^{2},
$$

where $\lambda_{1}$ is an arbitrary constant.

b) The equation

$$
X^{\prime \prime \prime}+5 X^{-1} X^{\prime} X^{\prime \prime}+\lambda_{1} X^{2} X^{\prime}=0
$$

leads to a Sundman symmetry of the form (4.11)-(4.12) with

$$
P=\left(c_{1}+c_{2} F^{4}\right)^{1 / 4}, \quad \Psi=F\left(c_{1}+c_{2} F^{4}\right)^{-1 / 4},
$$


where $\lambda_{1}$ and $c_{1}$ are arbitrary constants and $c_{2}$ is an arbitrary nonzero constant.

c) The equation

$$
X^{\prime \prime \prime}-X^{-1} X^{\prime} X^{\prime \prime}+\lambda_{1} X^{2} X^{\prime}=0
$$

leads to a Sundman symmetry of the form (4.11)-(4.12) with

$$
P=\left(c_{1}+c_{2} F^{2}\right)^{1 / 2}, \quad \Psi=F\left(c_{1}+c_{2} F^{2}\right)^{-1 / 2},
$$

where $\lambda_{1}$ and $c_{1}$ are arbitrary constants and $c_{2}$ is an arbitrary nonzero constant.

d) The equation

$$
X^{\prime \prime \prime}+X X^{\prime \prime}-7 X^{-1} X^{\prime} X^{\prime \prime}+8 X^{-2}\left(X^{\prime}\right)^{3}-2\left(X^{\prime}\right)^{2}+\lambda_{1} X^{2} X^{\prime}+\lambda_{0}\left(X^{8}-1\right)=0
$$

leads to a Sundman symmetry of the form (4.11)-(4.12) with

$$
P=F^{-1}, \quad \Psi=F^{2} \quad \text { or } \quad P=-F^{-1}, \quad \Psi=-F^{2},
$$

where $\lambda_{1}$ is an arbitrary constant and $\lambda_{0}$ is an arbitrary nonzero constant.

e) The equation

$$
X^{\prime \prime \prime}+X X^{\prime \prime}-7 X^{-1} X^{\prime} X^{\prime \prime}+8 X^{-2}\left(X^{\prime}\right)^{3}-2\left(X^{\prime}\right)^{2}+\lambda_{1} X^{2} X^{\prime}=0
$$

leads to a Sundman symmetry of the form (4.11)-(4.12) with

$$
P=c_{2}^{-1} F^{1-k}, \quad \Psi=c_{2} F^{k},
$$

where $\lambda_{1}$ is an arbitrary constant, $c_{2}$ is an arbitrary nonzero constant and $k$ is an arbitrary constant but $k \neq 1$.

f) The equation

$$
\begin{aligned}
X^{\prime \prime \prime} & +X X^{\prime \prime}-7 X^{-1} X^{\prime} X^{\prime \prime}+8 X^{-2}\left(X^{\prime}\right)^{3}-2\left(X^{\prime}\right)^{2} \\
& +\lambda_{1}\left(X^{4}+c_{2}^{-2}\right) X^{\prime}+\lambda_{0}\left(X^{8}-c_{2}^{-4}\right)=0
\end{aligned}
$$

leads to a Sundman symmetry of the form (4.11)-(4.12) with

$$
P=c_{2}^{-1} F^{-1}, \quad \Psi=c_{2} F^{2},
$$

where $\lambda_{0}$ and $\lambda_{1}$ are arbitrary constants and $c_{2}$ is an arbitrary nonzero constant.

A special Case: We consider Proposition 4.1 with $a_{3}=0$ and $a_{4}=0$ with the aim to find Sundman symmetries for this equation. Solving conditions (4.5)-(4.10) for this case we are led to the following

Corollary 4.1 Equation (4.2) with $a_{3}=a_{4}=0$, which can be transformed to

$$
X^{\prime \prime \prime}+a_{5}(X) X^{\prime \prime}+a_{2}(X)\left(X^{\prime}\right)^{2}+a_{1}(X) X^{\prime}+a_{0}(X)=0
$$

under the generalised Sundman transformation (1.1), admits a Sundman symmetry of the form (2.3) if and only if

$$
\begin{aligned}
& P(F)=c_{2}[\alpha F+\beta]^{-1}+c_{1} \\
& Q(F, G)=G[\alpha F+\beta]^{-3 / 2},
\end{aligned}
$$


where $c_{1}, \beta$ are arbitrary constants, $c_{2}, \alpha$ are arbitrary nonzero constants and $a_{5}, a_{2}, a_{1}$ and $a_{0}$ satisfy the following conditions:

$$
\begin{aligned}
& a_{5}(P(F))=(\alpha F+\beta)^{3 / 2} a_{5}(F) \\
& a_{2}(P(F))=-\frac{1}{c_{2} \alpha}(\alpha F+\beta)^{7 / 2} a_{2}(F)-\frac{1}{2 c_{2}}(\alpha F+\beta)^{5 / 2} a_{5}(F) \\
& a_{1}(P(F))=(\alpha F+\beta)^{3} a_{1}(F) \\
& a_{0}(P(F))=-c_{2} \alpha(\alpha F+\beta)^{5 / 2} a_{0}(F) .
\end{aligned}
$$

The Sundman symmetry of (4.2) with $a_{3}=a_{4}=0$ is therefore of the form

$$
\begin{aligned}
& F(\tilde{x}, \tilde{t})=c_{2}[\alpha F(x, t)+\beta]^{-1}+c_{1} \\
& G(\tilde{x}, \tilde{t}) d \tilde{t}=G(x, t)[\alpha F(x, t)+\beta]^{-3 / 2} d t,
\end{aligned}
$$

where conditions (4.16)-(4.19) must be satisfied.

We give some examples of equations with Sundman symmetries for Corollary 4.1.

Example 4.2: Firstly we consider

$$
X^{\prime \prime \prime}+\lambda X^{-3 / 2} X^{\prime}=0 .
$$

By Corollary 4.1 we find the corresponding Sundman symmetry

$$
F(\tilde{x}, \tilde{t})=F^{-1}(x, t), \quad G(\tilde{x}, \tilde{t}) d \tilde{t}=G(x, t) F^{-3 / 2} d t .
$$

With the identity transformation

$$
X=x, \quad T=t,
$$

that is $F(x, t)=x$ and $G=1$, we obtain a Sundman symmetry for

$$
\dddot{x}+\lambda x^{-3 / 2} \dot{x}=0,
$$

namely

$$
\tilde{x}=x^{-1}, \quad \tilde{t}=\int x^{-3 / 2} d t .
$$

We note that the first integral

$$
I_{1}=\ddot{x}-2 \lambda x^{-1 / 2}
$$

of (4.24) may be transformed to the first integral

$$
I_{2}=\frac{1}{2} \dot{x}^{2}-x \ddot{x}-2 \lambda x^{1 / 2}
$$

of (4.24). We remark that (4.24) is not linearisable to $X^{\prime \prime \prime}=0$ under a generalised Sundman transformation, as can easily be verified with the conditions given in [5]. 
As part of this example we also consider the Ansatz

$$
a_{5}=\lambda_{5} X^{m}, \quad a_{2}=\lambda_{2} X^{n}, \quad a_{1}=\lambda_{1} X^{p}, \quad m, n, p \in \mathcal{Q}, \quad \lambda_{1}, \lambda_{2}, \lambda_{3} \in \Re
$$

in equation (4.13). It follows by Corollary 4.1 that the corresponding equation (4.2) admits the Sundman symmetry (4.23) if and only if

$$
\lambda_{2}=-\frac{1}{4} \lambda_{5}, \quad m=-\frac{3}{4}, \quad n=-\frac{7}{4}, \quad p=-\frac{3}{2}
$$

that is (4.2) has the form

$$
X^{\prime \prime \prime}+\lambda_{5} X^{-3 / 4} X^{\prime \prime}-\frac{1}{4} \lambda_{5} X^{-7 / 4}\left(X^{\prime}\right)^{2}+\lambda_{1} X^{-3 / 2} X^{\prime}=0 .
$$

On the other hand

$$
X^{\prime \prime \prime}+\lambda X^{-7 / 4}\left(X^{\prime}\right)^{2}=0, \quad \lambda \in \Re,
$$

results in a Sundman symmetry of the form

$$
\begin{aligned}
& F(\tilde{x}, \tilde{t})=F^{-1}(x, t) \\
& G(\tilde{x}, \tilde{t}) d \tilde{t}=-G(x, t) F^{-3 / 2} d t
\end{aligned}
$$

for the corresponding equation (4.2). Note the minus sign in the last term and compare this Sundman symmetry with the Sundman symmetry (4.23)!

The third-order linearisable case: By Proposition 4.1 we have

Corollary 4.2: Every third-order ordinary differential equation which can be transformed to

$$
X^{\prime \prime \prime}=0
$$

under the generalised Sundman transformation (1.1) admits the Sundman symmetry (4.20)(4.21), that is

$$
\begin{aligned}
& F(\tilde{x}, \tilde{t})=c_{2}[\alpha F(x, t)+\beta]^{-1}+c_{1} \\
& G(\tilde{x}, \tilde{t}) d \tilde{t}=G(x, t)[\alpha F(x, t)+\beta]^{-3 / 2} d t,
\end{aligned}
$$

where $c_{1}, \beta$ are arbitrary constants and $c_{2}, \alpha$ are arbitrary nonzero constants.

Note that Corollary 4.2 corresponds to the observation of the Sundman symmetry for linearisable third-order equations in [5], where we now have a more general transformtion with arbitrary constants $c_{1}, c_{2}, \alpha, \beta$.

Example 4.3: We return to equation (1.4) discussed in the Introductory Example, namely

$$
\dddot{x}-x^{-1} \dot{x} \ddot{x}=0 .
$$


Our aim is to find the general solution for this equation from the particular solution

$$
x=t
$$

and the Sundman symmetry (4.27) - (4.28). For simplicity we let $\beta=0$. With $F=x^{2}$, $G=x$ and (4.29) the Sundman symmetry for (1.4), namely

$$
\begin{aligned}
& \tilde{x}^{2}=c_{2} \alpha^{-1} x^{-2}+c_{1} \\
& \tilde{x} d \tilde{t}=\alpha^{-3 / 2} x^{-2} d t,
\end{aligned}
$$

takes the form

$$
\tilde{x}^{2}=c_{2} \alpha^{-1} t^{-2}+c_{1}, \quad \tilde{x} d \tilde{t}=\alpha^{-3 / 2} t^{-2} d t .
$$

We insert $\tilde{x}$ from (4.30a) in (4.30b) and integrate to obtain

$$
\tilde{t}=\alpha^{-1} c_{2}^{-1 / 2}\left(\ln \left|\frac{c_{2}+c_{2}^{1 / 2}\left(c_{2}+c_{1} \alpha t^{2}\right)^{1 / 2}}{t}\right|-\ln \left|c_{3}\right|\right)
$$

$\left(\ln \left|c_{3}\right|\right.$ is a constant of integration) or by (4.30a) we can express $t$ in terms of $\tilde{t}$ and $\tilde{x}$, namely

$$
\frac{c_{2}}{t}=c_{3} \exp \left[\alpha c_{2}^{1 / 2} \tilde{t}\right]-c_{2}^{1 / 2} \alpha^{1 / 2} \tilde{x} .
$$

The transformation (4.30a) is equivalent to

$$
\frac{c_{2}^{2}}{t^{2}}=c_{2} \alpha \tilde{x}^{2}-c_{1} c_{2} \alpha
$$

which we insert in (4.32) to obtain

$$
\tilde{x}(\tilde{t})=\frac{1}{2}\left(c_{1} c_{2}^{1 / 2} c_{3}^{-1} \alpha^{1 / 2}\right) \exp \left[-\alpha c_{2}^{1 / 2} \tilde{t}\right]+\frac{1}{2}\left(c_{2}^{-1 / 2} c_{3} \alpha^{-1 / 2}\right) \exp \left[\alpha c_{2}^{1 / 2} \tilde{t}\right] .
$$

We now rename the constants as follows:

$$
\alpha c_{2}^{1 / 2} \equiv \beta_{1}, \quad \frac{1}{2} c_{1} c_{2}^{1 / 2} c_{3}^{-1} \alpha^{1 / 2} \equiv \beta_{2}, \quad \frac{1}{2} c_{2}^{-1 / 2} c_{3} \alpha^{-1 / 2} \equiv \beta_{3},
$$

and obtain the general solution of (1.4) (we drop the tilda), namely

$$
x(t)=\beta_{2} e^{-\beta_{1} t}+\beta_{3} e^{\beta_{1} t} .
$$

Example 4.4: Consider

$$
\dddot{x}=a_{1} x \ddot{x}+a_{2} \dot{x}^{2}+a_{3} x^{2} \dot{x}+a_{4} x^{4},
$$

where $a_{j}$ are constants. By the general conditions provided in [5] we find that the only equation of this type which linearises to (4.26) under a generalised Sundman transformation (1.1) is

$$
\dddot{x}=\lambda x \ddot{x}+2 \lambda \dot{x}^{2},
$$


where $\lambda$ is an arbitrary constant. In fact the generalised Sundman transformation that linearises $(4.34)$ to $(4.26)$ is

$$
\begin{aligned}
& X=\frac{\left(2 \lambda \alpha_{1} t+\beta_{1}\right) x+3 \alpha_{1}}{\left(2 \lambda \alpha_{2} t+\beta_{2}\right) x+3 \alpha_{2}} \\
& d T=3^{3 / 2}\left[\left(2 \lambda \alpha_{2} t+\beta_{2}\right) x+3 \alpha_{2}\right]^{-3 / 2} d t .
\end{aligned}
$$

Note that $\alpha_{1}, \alpha_{2}, \beta_{1}, \beta_{2}$ are arbitrary constants, subject to the constraint

$$
\beta_{1} \alpha_{2}-\alpha_{1} \beta_{2} \neq 0,
$$

that is

$$
\frac{\left(2 \lambda \alpha_{1} t+\beta_{1}\right) x+3 \alpha_{1}}{\left(2 \lambda \alpha_{2} t+\beta_{2}\right) x+3 \alpha_{2}} \neq \text { constant. }
$$

We now use (4.36)-(4.37) to obtain two independent first integrals of (4.35). We transform the two first integrals of (4.26) which provide one $t$-independent and one $t$-dependent first integral for (4.35), namely

$$
\begin{aligned}
& I_{1}=x \ddot{x}-\frac{1}{2} \dot{x}^{2}-\lambda x^{2} \dot{x} \\
& I_{2}=\lambda t \dot{x}^{2}+\left(5 \lambda x+2 \lambda^{2} x^{2} t\right) \dot{x}-(2 \lambda t x+3) \ddot{x}-\frac{2}{3} \lambda^{2} x^{3} .
\end{aligned}
$$

By Corollary 4.2 the Sundman symmetry of (4.35) is

$$
\begin{aligned}
& F(\tilde{x}, \tilde{t})=c_{2}[\alpha F(x, t)+\beta]^{-1}+c_{1} \\
& G(\tilde{x}, \tilde{t}) d \tilde{t}=G(x, t)[\alpha F(x, t)+\beta]^{-3 / 2} d t,
\end{aligned}
$$

where

$$
\begin{aligned}
& F(x, t)=\frac{\left(2 \lambda \alpha_{1} t+\beta_{1}\right) x+3 \alpha_{1}}{\left(2 \lambda \alpha_{2} t+\beta_{2}\right) x+3 \alpha_{2}} \\
& G(x, t)=3^{3 / 2}\left[\left(2 \lambda \alpha_{2} t+\beta_{2}\right) x+3 \alpha_{2}\right]^{-3 / 2}
\end{aligned}
$$

with the conditions on the constants as given in Corollary 4.2 and above.

We further remark that equation (4.35) is not listed in Chazy's paper [1]. This is explained by the fact that (4.35) does not pass the Painlevé test. Besides the Fuchs indices -1 and $4,(4.35)$ has a noninteger Fuchs index namely $3 / 2$ [2]. It seems that the linearisation of (4.35) cannot be accomplished by the use of other standard transformations [9]. We also mention that we were not able to obtain the general solution of (4.35) from the linearisation for the reason that the combination of the two integrals (4.38) and (4.39) leads to a first order Abel's equation of the second kind which can be reduced to an Abel's equation of the first kind, neither of which we were able to solve.

As part of this example we investigate the invariance of (4.34) under the generalised Sundman transformation (1.1) with the following result: The Chazy class of equations (4.34) remains invariant under the generalised Sundman transformation

$$
\tilde{x}(\tilde{t})=F(x, t), \quad d \tilde{t}=G(x, t) d t
$$


if and only if (4.34) takes the form

$$
\dddot{x}=a_{1} x \ddot{x}+a_{2} \dot{x}^{2}-\frac{a_{1}}{2}\left(\frac{a_{2}}{3}+\frac{a_{1}}{2}\right) x^{2} \dot{x}+\frac{a_{1}^{2}}{48}\left(\frac{a_{2}}{3}+\frac{a_{1}}{2}\right) x^{4}
$$

and (4.40) takes the form

$$
\begin{aligned}
& \tilde{x}(\tilde{t})=(\alpha t+\beta)^{2} x+\frac{12 \alpha}{a_{1}}(\alpha t+\beta) \\
& d \tilde{t}=(\alpha t+\beta)^{-2} d t,
\end{aligned}
$$

where $a_{1}$ and $\alpha$ are arbitrary nonzero constants, $a_{2}$ and $\beta$ are arbitrary constants and

$$
\frac{a_{2}}{a_{1}} \neq \frac{1}{3} \text {. }
$$

For example let $\alpha=1$ and $\beta=0$. Then (4.41) admits the discrete symmetry

$$
\tilde{x}=t^{2} x+\frac{12}{a_{1}} t, \quad \tilde{t}=-\frac{1}{t} .
$$

We remark that the discrete transformation (4.42) was also obtained in [6] for $a_{1}=2$ and $a_{2}=36 \lambda-3$ ( $\lambda$ an arbitrary constant) by the use of the Lie point symmetry algebra. We further remark that for arbitrary constants $a_{1}$ and $a_{2}$ the maximum Lie point symmetry generators for (4.41) are

$$
\begin{aligned}
& Z_{0}=\frac{\partial}{\partial t}, \quad Z_{1}=-t \frac{\partial}{\partial t}+x \frac{\partial}{\partial x} \\
& Z_{2}=-\frac{a_{1} t^{2}}{12} \frac{\partial}{\partial t}+\left(1+\frac{a_{1} x t}{6}\right) \frac{\partial}{\partial x}
\end{aligned}
$$

whereas the maximum Lie point symmetry generators for (4.34) are

$$
Z_{0}=\frac{\partial}{\partial t}, \quad Z_{1}=-t \frac{\partial}{\partial t}+x \frac{\partial}{\partial x}
$$

for arbitrary constants $a_{1}, a_{2}, a_{3}$ and $a_{4}$.

In the final example we consider an inverse problem: We start with a first integral of the order two, write the third-order equation which admits this first integral, construct a Sundman symmetry for the resulting equation and use this Sundman symmetry to find a second independent first integral. We then introduce a generalised Sundman transformation to obtain a class of third-order equations all of which admit a Sundman symmetry and two first integrals.

Example 4.5: Consider

$$
I_{1}=X^{\prime \prime}+\lambda X^{-1}\left(X^{\prime}\right)^{2},
$$

where $\lambda$ is an arbitrary constant with $\lambda \notin\{-3 / 2,-1 / 2\}$. By differentiating (4.43) we obtain the equation which admits (4.43) as a first integral, namely the equation

$$
X^{\prime \prime \prime}+2 \lambda X^{-1} X^{\prime} X^{\prime \prime}-\lambda X^{-2}\left(X^{\prime}\right)^{3}=0
$$


By Proposition 4.1 equation (4.44) leads to a Sundman Symmetry of the form (2.3) with

$$
P=c_{1} F^{-1}, \quad Q=c_{2} G F^{-3 / 2-\lambda},
$$

where $c_{1}$ and $c_{2}$ are arbitrary nonzero constants. With the identity transformation

$$
X=x, \quad T=t,
$$

that is $F=x$ and $G=1$, equation (4.44) transforms to itself, i.e.

$$
\dddot{x}+2 \lambda x^{-1} \dot{x} \ddot{x}-\lambda x^{-2} \dot{x}^{3}=0 .
$$

Thus corresponding to this identity transformation the Sundman symmetry of (4.44) (or equivalently (4.46)) is

$$
\tilde{X}=X^{-1}, \quad d \tilde{T}=X^{-3 / 2-\lambda} d T .
$$

We transform the first integral (4.43) of (4.44) by (4.47) to obtain the second independent first integral of (4.44), namely

$$
I_{2}=X^{2 \lambda+1}\left(X^{\prime \prime}-\frac{1}{2} X^{-1}\left(X^{\prime}\right)^{2}\right) .
$$

We now consider the generalised Sundman transformation

$$
X=x^{n}, \quad d T=x^{m} d t, \quad n \in \mathcal{Q} \backslash 0, \quad m \in \mathcal{Q}
$$

for (4.44) to obtain

$$
\dddot{x}+p x^{-1} \dot{x} \ddot{x}+q x^{-2} \dot{x}^{3}=0,
$$

where $p$ and $q$ are constants which are related to $m$ and $n$ as follows:

$$
\begin{aligned}
& n=\frac{\sqrt{(p-1)^{2}-8 q}}{2 \lambda+1} \\
& m=\frac{1}{4}\left(\frac{2 \lambda+3}{2 \lambda+1}\right) \sqrt{(p-1)^{2}-8 q}-\frac{1}{4}(p+3) .
\end{aligned}
$$

Equation (4.48) admits the two first integrals

$$
\begin{aligned}
& I_{1}=x^{n-2 m-1}\left[\ddot{x}+(n-m+\lambda n-1) x^{-1} \dot{x}^{2}\right] \\
& I_{2}=x^{2 n(\lambda+1)-2 m-1}\left[\ddot{x}-\frac{1}{2}(2 m-n+2) x^{-1} \dot{x}^{2}\right]
\end{aligned}
$$

and the Sundman symmetry

$$
\tilde{x}=x^{-1}, \quad \tilde{t}=\int x^{2 m-\lambda-3 n / 2} d t .
$$

For example let $\lambda=1 / 2, p=-1$ and $q=-3 / 2$. Then

$$
\dddot{x}-x^{-1} \dot{x} \ddot{x}-\frac{3}{2} x^{-2} \dot{x}^{3}=0
$$


is transformed to

$$
X^{\prime \prime \prime}+X^{-1} X^{\prime} X^{\prime \prime}-\frac{1}{2} X^{-2}\left(X^{\prime}\right)^{3}=0
$$

by the generalised Sundman transformation

$$
X=x^{2}, \quad d T=x^{3 / 2} d t
$$

and admits the Sundman symmetry

$$
\tilde{x}=x^{-1}, \quad \tilde{t}=\int x^{-1 / 2} d t
$$

\section{Concluding remarks}

In this article we have shown that Sundman symmetries are admitted by a large class of second-order and third-order ordinary differential equations, even where these equations are not linearisable by a generalised Sundman transformation. We presented several Propositions by which one is able to construct Sundman symmetries of nonlinear ordinary differential equations and we gave many examples of equations and their Sundman symmetries which follow from these Propositions. The variety of equations which admit Sundman symmetries is quite remarkable. In some cases there exist even infinitely many nontrivial Sundman symmetries. It is currently not clear to us what the connections are, if any, between the Sundman symmetries and the Lie (point or contact) symmetries of the equations, especially due to the nonlocal structure of the Sundman symmetries.

We have shown that the Sundman symmetries can successfully be applied to transform first integrals to new first integrals which may lead to the general solution of the corresponding equations. We have also shown that generalised Sundman symmetries can be used to map special solutions to general solutions. An interesting example is the linearisation of the Chazy-type equation (4.35), which shows that the generalised Sundman transformation does not preserve the Painlevé property nor does it preserve the Lie symmetry structure of the equations.

It would be useful to 'invert' the conditions on the $\Lambda$ 's given in each Proposition, to obtain necessary and sufficient conditions for a given second- or third-order ordinary differential equation for which the equations are transformable under a generalised Sundman transformation to the autonomous equations given in the Propositions and hence provide the equations with a Sundman symmetry. This was done for the case of linearisable third-order ordinary differential equations in [5]. For a general classification of equations and their Sundman symmetries it is necessary to obtain the analogue results for each Proposition given here.

\section{Acknowledgements}

We thank Robert Conte, Peter Leach and Clara Nucci for their comments and remarks concerning this work which led to the current revised version of our preprint. 


\section{References}

[1] Chazy J, Sur les équations différentielles du troisième ordre et d'ordre supérieur dont l'intégrale générale a ses points critiques fixes, Acta Mathematica 34 (1911), 317-385.

[2] Conte R, private communication, January 28, 2004.

[3] Duarte L G S, Moreira I C \& Santos FC, Linearisation under nonpoint transformations $J$ Phys A: Math Gen 27 (1994), L739-L743.

[4] Euler N and Leach P G L, First integrals and reduction of a class of nonlinear higher order ordinary differential equations, J Math. Anal. Appl. 287(2) (2003), 473-486.

[5] Euler N, Wolf T, Leach P G L and Euler M, Linearisable third-order ordinary differential equations and generalised Sundman transformations: The Case $X^{\prime \prime \prime}=0$, Acta Appl. Math. 76 (2003), 89-115.

[6] Hydon P E, Discrete point symmetries of ordinary differential equations, R. Soc. Lond. Proc. Ser. A Math. Phys. Eng. Sci. 454 (1998), 1961-1972.

[7] Hydon P E, Discrete symmetries of differential equations, Contemporary Mathematics 285 (2001), 61-70.

[8] Laine-Pearson F E and Hydon P E, Classification of discrete symmetries of ordinary differential equations, Stud. Appl. Math. 111 (2003), 269-299.

[9] Leach P G L, private communication, January 14, 2004.

[10] Steeb W-H, Invertible Point Transformations and Nonlinear Differential Equations, World Scientific, Singapore, 1993. 